\title{
Nuclear analytical techniques for nanotoxicology studies
}

\author{
By Z. Y. Zhang*, Y. L. Zhao and Z. F. Chai \\ Key Laboratory of Nuclear Analytical Techniques, Key Lab for Biomedical Effects of Nanomaterials \& Nanosafety, \\ Institute of High Energy Physics, Chinese Academy of Sciences, Beijing 100049, China
}

(Received December 2, 2009; accepted in revised form November 12, 2010)

\section{Nuclear analytical techniques / Nanomaterials / \\ Nanotoxicology / Characterization / \\ Quantitative detection}

Summary. With the rapid development of nanotechnology and its applications, a wide variety of nanomaterials are now used in commodities, pharmaceutics, cosmetics, biomedical products, and industries. The potential interactions of nanomaterials with living systems and the environment have attracted increasing attention from the public, as well as from manufacturers of nanomaterial-based products, academic researchers and policymakers. It is important to consider the environmental, health and safety aspects at an early stage of nanomaterial development and application in order to more effectively identify and manage potential human and environmental health impacts from nanomaterial exposure. This will require research in a range of areas, including detection and characterization, environmental fate and transport, ecotoxicolgy and toxicology. Nuclear analytical techniques (NATs) can play an important role in such studies due to their intrinsic merits such as high sensitivity, good accuracy, high space resolution, ability to distinguish the endogenous or exogenous sources of materials, and ability of in situ and in vivo analysis. In this paper, the applications of NATs in nanotoxicological and nanoecotoxicological studies are outlined, and some recent results obtained in our laboratory are reported.

\section{Introduction}

Nanotechnologies have been widely recognized as having huge potential to affect drug development, water decontamination, development of information and communication technologies, as well as production of stronger and lighter materials. With the rapid development of nanotechnology and its applications, a wide variety of nanomaterials are now used in clothing, electronic devices, cosmetics, pharmaceuticals and other biomedical products. While possessing more novel and unique properties than bulk materials (smaller size, larger surface area and higher reactivity), nanoparticles also have unpredictable impacts on human health and environment. The entrance into and interaction of nanomaterials in the human body have generated intense scientific curiosity, attracting increasing concerns from the public, nanomaterial-based industries, academia, and governments worldwide [1].

\footnotetext{
*Author for correspondence (E-mail: zhangzhy@ihep.ac.cn).
}

Nanotoxicology is emerging as an important sub-discipline of nanotechnology. It refers to the study of the interactions of nanostructures (e.g. size, shape, surface chemistry, composition, and aggregation) with biological systems with an emphasis on elucidating the relationship between the physical and chemical properties of nanomaterials with induction of toxic biological responses [2]. The importance of nanotechnology to the economy and our future well-being is beyond debate, but its potential adverse impacts need to be studied along the same lines. A discipline of nanotoxicology would make an important contribution to the development of a sustainable and safe nanotechnology.

Nanotoxicology relies on many analytical methods for the characterization of nanomaterials as well as their impacts on in vitro and in vivo function. In this case, nuclear analytical techniques (NATs) can play an important role in characterization of nanomaterials and quantitative detection of nanomaterials in biological and environmental samples. The definition of nuclear analytical techniques is flexible [3], here we define NATs as the analytical methods based on nuclear parameters, nuclear reactions, nuclear effects, nuclear radiations, nuclear spectroscopy and nuclear facilities.

\section{Characterization of nanomaterials by NATs}

In nanotoxicology studies, proper characterization of test materials is important to ensure reproducible results, and to provide the basis of understanding the properties of nanoparticles which determine their biological effects. Because key parameters affecting biological activities of nanoparticles are largely unknown at this point, the characterization of test materials must be comprehensive and broad in scope. There are a number of fundamental properties that researchers in the field generally agree must be addressed, these include size and shape, state of dispersion, physical and chemical properties, surface area, and surface chemistry [4].

Various NATs can be applied to study physical and chemical characteristics of nanomaterials, which mainly include the synchrotron radiation (SR) and neutron source based approaches (Table 1).

SR-based techniques have been proven to be highly beneficial in many fields of research on the characterization of nanomaterials $[5,6]$. SR sources produce an intense X-ray emission with total fluxes and flux densities several orders of magnitude greater than conventional X-ray tubes. The 
Table 1. NATs used in characterization of nanomaterials. ${ }^{a}$

Characteristics of nanomaterials NATs

Chemical composition

Crystal structure

Shape

Size distribution

Surface chemistry

a: Abbrevations: SR-XPS, synchrotron radiation X-ray photoeletron spectroscopy; SR-XRF, synchrotron radiation X-ray fluoresence, NAA, neutron activation analysis; SR-XRD, synchrotron radiation X-ray diffraction; SANS, small angle neutron sacttering; XAS, X-ray absorption spectroscopy.

combination of unique and controllable source properties enables a broad range of experimental techniques. Conventional laboratory techniques such as XPS, XRD and XRF, may be performed much more rapidly with enhanced signalto-noise ratios. Coupled with high performance monochromation, the high brightness results in great improvements in spectral resolution [7].

NAA is one of the most sensitive analytical techniques for multi-element determination. Unlike other traditional methods, NAA normally does not require any sample pretreatment and is especially suited for solid materials that are difficult to dissolve. The low availability of an irradiation facility is a major limitation of NAA, along with the running cost and radiation protection. ICP-MS is widely acknowledged as the premier technique for trace metals analysis. It offers exceptional sensitivity and excellent accuracy along with multi-element and isotope ratio measurement capabilities. Before ICP-MS determination, samples must be pretreated and prepared into solution form. However, standard sample pretreatment procedures are unavailable for some special materials, such as carbon nanotubes (CNTs). Ge et al. [8] established suitable sample pretreatment procedures for ICP-MS analysis of CNTs using NAA as a standard quantification method. CNTs were pretreated with the following methods, dry ashing coupled with acid extraction, wet digestion, a combination of dry ashing with acid digestion, and microwave-assisted acid digestion, then analyzed by ICP-MS. The results indicate that a combination of dry ashing with wet digestion and microwave digestion can effectively remove metals from CNTs. After two pretreatment procedures, the analytical results of ICP-MS were closely matched with those of the NAA. These methods have been proposed as ISO standard pretreatment methods of metal impurities determination in CNTs using ICP-MS.

\section{Detection of nanomaterials in biological and environmental samples by NATs}

Determination of nanomaterials in organisms or environmental matrix is frequently required in toxicological and ecotoxicological studies of nanomaterials. It is a new challenge for modern analytical techniques. Some special nanomaterials such as quantum dots and magnetic nanocrystals can be determined using their fluorescent or magnetic properties $[9,10]$. Trace metal analytical methods (ICP-MS, ICP-
AES, AAS, etc.) are generally applied in detection of metalcontaining nanomaterials. NATs SR-XRF and scanning proton microprobe (SPM) are characterized of multi-elements, high sensitivity, microanalysis and positional analysis. Wang et al. [11] studied the translocation of inhaled $\mathrm{TiO}_{2}$ nanoparticles along olfactory nervous system to brain by SR-XRF. Mice were exposed to nano- $\mathrm{TiO}_{2}$ by intranasal administration for one month. Titanium distributions in brain sections were analyzed. The results show that nano- $\mathrm{TiO}_{2}$ could enter the olfactory nerve layer, granular cell layer of olfactory bulb, olfactory ventricle and further the hippocampus, thalamus, and $\mathrm{CA}_{3}$ area of the brain through the olfactory nervous system. In another study, the transport of intranasally instilled fine $\mathrm{Fe}_{2} \mathrm{O}_{3}$ particles into the brain was investigated [12]. The micro-distribution map of iron in the olfactory bulb and brain stem shows an obvious increase of Fe contents in the olfactory nerve and the trigeminus of brain stem, suggesting that $\mathrm{Fe}_{2} \mathrm{O}_{3}$ particles were possibly transported via uptake by sensory nerve endings of the olfactory nerve and trigeminus.

Nevertheless, the above trace metal analytical methods are not able to distinguish between nanomaterials and the backgrounds. If the background value of the metal is relatively high, the results will be severely interfered. This problem can be solved by using isotopic tracer techniques, especially radiotracer techniques. Radiotracer techniques offer many advantages over traditional methods, such as high sensitivity, good accuracy, time savings, and the ability to distinguish between the endogenous and exogenous sources of materials [13]. In many cases they are indispensable.

Radiolabeling methods of carbon nanomaterials have been extensively studied, and labeled products of almost all kinds of carbon nanomaterials have been reported [14]. But few radiolabeled metallic and metal oxide nanoparticles have been reported because of lack of labeling methods. Radioactive metallic and metal oxide nanomaterials can be prepared by irradiation of stable nanomaterials in a nuclear reactor. However, the reliability of this method needs further confirmation. First, the irradiation damage on the nanostructure is inevitable. Second, the activated atoms may be released from the crystal lattice by the Szilard-Chalmers effect. So measured radioactivity might not be from the nanomaterials themselves [14]. The best method for radiolabeling nanomaterials is the incorporation of tracers under nanoparticle production. Thus, the prepared radiolabeled nanoparticles will share the same characteristics with the stable ones. Recently, we synthesized radioactive ceria nanoparticles using a precipitation method. Radioactive ${ }^{141} \mathrm{Ce}$ was produced by thermal neutron bombardments of stable $\mathrm{CeO}_{2}$ in a swimming pool reactor. After irradiation, ${ }^{141} \mathrm{CeO}_{2}$ was chemically transformed into ${ }^{141} \mathrm{Ce}\left(\mathrm{NO}_{3}\right)_{3}$ with $\mathrm{H}_{2} \mathrm{O}_{2}$ and $\mathrm{HNO}_{3}$. Then, ${ }^{141} \mathrm{Ce}\left(\mathrm{NO}_{3}\right)_{3}$ solution was reacted with an organic base hexamethylenetetramine. Finally, radioactive ${ }^{141} \mathrm{CeO}_{2}$ nanoparticles were obtained. The particle sizes of nano- ${ }^{141} \mathrm{CeO}_{2}$ can be controlled by changing the organic base concentrations. Higher concentration will lead to smaller size nanoparticles. A series of studies on the behavior of nano-ceria in aquatic environment, plants, and animals have been carried out in our laboratory.

To get an initial estimate of the environmental behavior of nanomaterials, the distribution and accumulation of 
ceria nanoparticles in simulated aquatic ecosystems which included aquatic plant, shellfish, fish, water, and sediment were investigated [15]. The results indicate that the clearance of ceria nanoparticles from water was fast. After $3 \mathrm{~d}$, more than $99.5 \%$ of total nano-ceria was eliminated from water. Not surprisingly, most nano-ceria was deposited on the sediments. The distribution and accumulation characteristics of nano-ceria in various aquatic organisms were different. Ceratophyllum demersum showed a high ability of accumulation of nano-ceria from water. In the cases of fish and snail, both the accumulation and elimination of nanoceria were fast. The total recovery of the experiment is about $64 \%$. It should be noted that other factors, such as turbulent mixing and bioturbation, which were not considered in this study, can complicate this simple picture. Nanomaterials may stay in the water-column for a longer time than the present results and therefore aquatic organisms are expected to be the main sinks and receptors of nanomaterials in surface waters.

Plants are an important component in the ecological system and may serve as a potential pathway for nanomaterial transport and a route for bioaccumulation into the food chain. However, the knowledge about the interactions of plants with nanoparticles is still scarce. We exposed cucumber and wheat plants to nano- ${ }^{141} \mathrm{CeO}_{2}$ suspension for $7 \mathrm{~d}$. The plants were separated into shoots and roots, radio activities of the samples were measured. It was found that nanoceria could be transported from roots to shoots. Translocation factors (defined as content ratio of shoot to root) of nano-ceria in plants were extremely low, about $10^{-3}-10^{-4}$. Cucumber plants showed a higher ability of accumulation and transportation ceria than wheat. An autoradiograph of ceria nanoparticles in cucumber leave showed that ceria

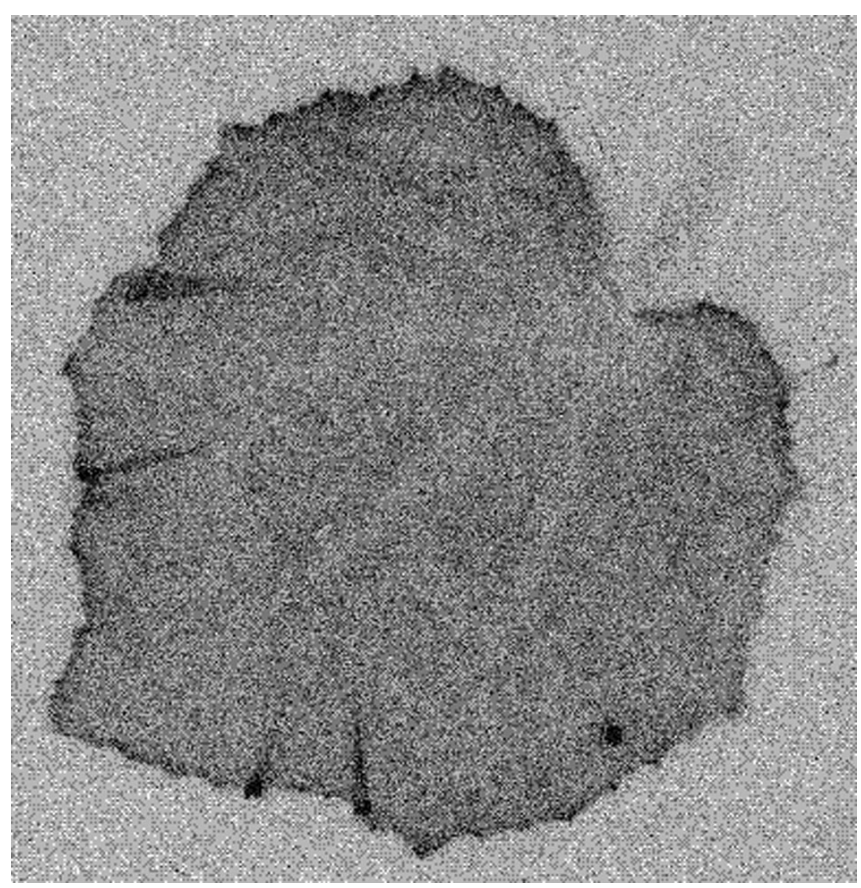

Fig. 1. Autoradiograph of nano- ${ }^{141} \mathrm{CeO}_{2}$ in a cucumber leaf. Cucumber plants were treated with $20 \mathrm{mg} / \mathrm{L}$ radioactive nano-ceria suspensions for $14 \mathrm{~d}$; autoradiography was performed by placing the leaves in contact with an imaging plate for $3 \mathrm{~d}$. nanoparticles were significantly accumulated along the margins of the leaves (Fig. 1).

Previous studies showed that nanoparticles could pass through the alveolar-capillary barrier and eventually cause adverse effects on cardiorespiratory function and blood coagulation, as well as on functions of the central nervous system [16]. Therefore, it is essential to study the absorption, distribution, metabolism, and excretion behaviors of inhaled nanomaterials in vivo, to understand the underlying mechanisms. The pulmonary deposition and translocation of nano- ${ }^{141} \mathrm{CeO}_{2}$ in rats after intratracheal instillation were studied [17]. It was found that about $63.9 \%$ of delivered nano-ceria remained in lung by $28 \mathrm{~d}$ postexposure, and the left part of given dose was mainly contained in feces. The results of whole-body distribution demonstrated that the translocation of nano-ceria from the respiratory epithelium towards circulation and subsequent systemic accumulation did take place after the nano-ceria was deposited in the alveolus; liver, spleen, bone and blood were the targets of extrapulmonary redistribution. Though occurred in a quite slow way, penetration to body circulations instead of phagocytosis by alveolar macrophages became the major route for the elimination of nano-ceria from lung at the end of the test period, and the alteration might attribute to the changes in dispersion of nano-ceria in lung.

Due to their larger surface energies, nanoparticles tend to be more chemically reactive and thus to undergo transformation more readily than larger particles, and this property causes them to exist as various species in vivo or in vitro [6]. Therefore, characterizing the chemical species of nanoparticles, which are often changed by biological systems before or after they act on target tissues, is crucial to elucidating the toxic mechanisms. Synchrotron radiation XAS can provide information on local electronic and molecular structure around the atom of interest with sub-angstrom spatial resolution and ppm detection limits [18].

XAS is typically separated into the X-ray absorption near-edge structure (XANES) region, which is the region
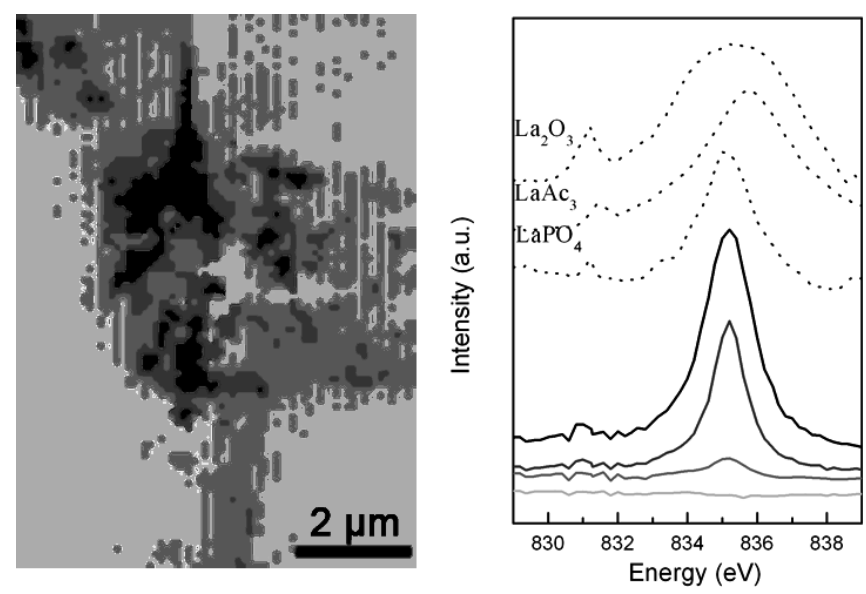

Fig. 2. (a) Composite map of La-components in a cucumber root section with $1 \mu \mathrm{m}$ thickness derived from an STXM La $3 d$ edge stack analysis. Black, dark grey, and grey = high, middle, and low-content La regions. Light grey $=$ non-La regions. Cucumber plants were treated with $2000 \mathrm{mg} / \mathrm{L} \mathrm{La}_{2} \mathrm{O}_{3} \mathrm{NPs}$ for $5 \mathrm{~d}$. (b) $\mathrm{La} 3 d$ spectra extracted from the image sequence, compared to the reference spectra $\left(\mathrm{LaPO}_{4}\right.$, $\mathrm{La}_{2} \mathrm{O}_{3}$, and $\left.\mathrm{La}(\mathrm{Ac})_{3}\right)$, showing that La spectra can be matched very well with the spectrum of $\mathrm{LaPO}_{4}$. 
up to about $50 \mathrm{eV}$ above the elemental absorption edge, and the extended X-ray absorption fine structure (EXAFS) region, which extends from the XANES region up to $1000 \mathrm{eV}$ or so above the absorption edge [6]. The XANES spectrum gives information about oxidation state, covalence, molecular symmetry of the site, and thereby coordination number. The EXAFS provides direct, local structural information about the atomic neighborhood of the element being probed. Using XANES, Wang et al. [12] demonstrated chemical transformation of fine $\mathrm{Fe} 2 \mathrm{O} 3$ particles in brain tissues of mice after intranasal exposure.

Synchrotron-based scanning transmission X-ray microscopy (STXM) techniques coupled with XANES can be used to analyze thin samples in situ with a spatial resolution of better than $30 \mathrm{~nm}$, with no need for prior chemical extraction or staining. The results of a previous study show that $\mathrm{La}_{2} \mathrm{O}_{3}$ NPs could inhibit root elongation of cucumber plants. But the underlying mechanism is unknown [20]. Recently, we investigate the biotransformation of $\mathrm{La}_{2} \mathrm{O}_{3}$ NPs in cucumber plants using STXMXANES. In the experiment, $\mathrm{La}_{2} \mathrm{O}_{3}$ NPs were transformed to needle-like $\mathrm{LaPO}_{4}$ nanoclusters in the intercellular spaces and middle lamellas of the cucumber roots (Fig. 2). This was similar to the behavior $\mathrm{La}^{3+}$ ion in plant roots. It was speculated that the phytotoxicity of $\mathrm{La}_{2} \mathrm{O}_{3} \mathrm{NPs}$ might be related to the dissolution of NPs at the nano-bio interface induced by the organic acids excreted from root cells.

\section{Conclusions}

Using nuclear analytical techniques, we have:

(1) Established standard sample preparation methods for chemical impurity analysis of CNTs;

(2) Confirmed that nano- and fine particles could be transferred via the secondary and tertiary olfactory pathways to reach most parts of brain;

(3) Quantitatively demonstrated the translocation of nanoceria from the respiratory epithelium towards circulation and proved that subsequent systemic accumulation did take place;

(4) Obtained information on accumulation and distribution characteristics of nanoparticles in simulated aquatic systems;

(5) Demonstrated the distribution characteristics and biotransformation of NPs in plants.

In further studies, we will focus on mechanisms of biological effects of nanomaterials. One of the major difficulties in such studies is how to characterize nanomaterials in vivo. Undoubtedly, NATs will continue to play a key role.

Acknowledgment. This work was financially supported by National Natural Science Foundation of China (10875136, 10905062, 11005118), and the Ministry of Science and Technology of China (2011CB933400).

\section{References}

1. Zhao, Y. L., Nalwa, H. S.: Nanotoxicology - Interactions of Nanomaterials with Biological Systems. American Scientific Publishers, Valencia, CA (2006), p. V.

2. Fischer, H. C., Chan, W. C.: Nanotoxicity: the growing need for in vivo study. Curr. Opin. Biotechnol. 18, 565 (2007).

3. Pounds, J. G., Jones, K. W.: Preface. Biol. Trace Elem. Res. 13, n5 (1987).

4. Powers, K. W., Brown, S. C. Krishna, V. B., Wasdo, S. C., Moudgil, B. M., Stephen, M. R.: Research strategies for safety evaluation of nanomaterials. Toxicol. Sci. 90, 296 (2006).

5. Adams, F., Van Vaecka, L., Barrett, R.: Advanced analytical techniques: platform for nano materials science. Spectrochim. Acta B 60, 13 (2005).

6. Wang, B., Wang, Z., Feng, W., Wang, M., Hu, Z., Chai, Z., Zhao, Y.: New methods for nanotoxicology: synchrotron radiationbased techniques. Anal. Bioanal. Chem. 398, 667 (2010).

7. Kempson, I. M., Kirkbride, K. P., Skinner, W. M., Coumbaros, J.: Applications of synchrotron radiation in forensic trace evidence analysis. Talanta 67, 286 (2005).

8. Ge, C. C., Lao, F., Li, W., Li, Y. F., Chen, C. C., Qiu, Y., Mao, X. Y., Li, B., Chai, Z. F., Zhao, Y. L.: Quantitative analysis of metal impurities in carbon nanotubes: efficacy of different pretreatment protocols for ICP-MS spectroscopy. Anal. Chem. 80, 9426 (2008).

9. Karabanovas, V., Zakarevicius, E., Sukackaite, A., Streckyte, G., Rotomskis, R.: Examination of the stability of hydrophobic (CdSe)ZnS quantum dots in the digestive tract of rats. Photochem. Photobiol. Sci. 7, 725 (2008).

10. Zhu, H., Han, J., Xiao, J. Q., Jin, Y.: Uptake, translocation, and accumulation of manufactured iron oxide nanoparticles by pumpkin plants. J. Environ. Monit. 10, 713 (2008).

11. Wang, J. X., Chen, C. Y., Sun, J., Yu, H. W., Li, Y. F., Li, B., Xing, L., Huang, Y. Y., He, W., Gao, Y. X., Chai, Z. F., Zhao, Y. L.: Translocation of inhaled $\mathrm{TiO}_{2}$ nanoparticles along olfactory nervous system to brain studied by synchrotron radiation X-ray fluorescence. High Energy Phys. Nucl. Phys. 29(Suppl.), 76 (2009).

12. Wang, B., Feng, W. Y., Wang, M., Shi, J. W., Zhang, F., Ouyang, H., Zhao, Y. L., Chai, Z. F., Huang, Y. Y., Xie, Y. N., Wang, H. F., Wang, J.: Transport of intranasally instilled fine $\mathrm{Fe}_{2} \mathrm{O}_{3}$ particles into the brain: micro-distribution, chemical states, and histopathological observation. Biol. Trace Elem. Res. 118, 233 (2007).

13. Zhang, Z. Y., Chai, Z. F.: Isotopic tracer studies of chemical behavior of rare earth elements in environmental and biological sciences. Radiochim. Acta 92, 355 (2004).

14. Zhang, Z. Y., Zhao, Y. L., Chai, Z. F.: Applications of radiotracer techniques for the pharmacology and toxicology studies of nanomaterials. Chin. Sci. Bull. 54, 173 (2009).

15. Lu, K., Zhang, Z. Y., He, X., Ma, Y. H., Zhou, K. B., Zhang, H. F., Bai, W., Ding, Y. Y., Wu, Z. Q., Zhao, Y. L., Chai, Z. F.: Bioavailability and distribution and of ceria nanoparticles in simulated aquatic ecosystems, quantification with a radiotracer technique. J. Nanosci. Nanotechnol. 10, 8658 (2010).

16. Kreyling, W., Semmler-Behnke, M., Moller, W.: Ultrafine particlelung interactions: does size matter? J. Aerosol Med. 19, 74 (2006).

17. He, X., Zhang, H. F., Ma, Y. H., Bai, W., Zhang, Z. Y., Lu, K., Ding, Y. Y., Zhao, Y. L., Chai, Z. F.: Lung deposition and extrapulmonary translocation of nano-ceria after intratracheal instillation, Nanotechnology 21, 285103 (2010).

18. Bertsch, P. M., Hunter, D. B.: Applications of synchrotron-based X-ray microprobes. Chem. Rev. 101, 1809 (2001).

19. Petibois, C., Guidi, M. C.: Bioimaging of cells and tissues using accelerator-based sources. Anal. Bioanal. Chem. 391, 1599 (2008).

20. Ma, Y., Kuang, L., He, X., Bai, W., Ding, Y., Zhang, Z., Zhao, Y., Chai, Z.: Effects of rare earth oxide nanoparticles on root elongation of plants. Chemosphere 78, 273 (2010). 